\title{
La esfera pública en la era de la hipermediación algorítmica: noticias falsas, desinformación y la mercantilización de la conducta
}

\author{
Sandra Álvaro \\ Investigadora y productora cultural \\ sandraalvaro@enlaciutat.es \\ https://orcid.org/0000-0001-5243-9814
}

\section{The public sphere in the epoch of the algorithmic hyper-mediation: Fake News, disinformation, misinformation and the commodification of behaviour}

\begin{abstract}
RESUMEN
La web y especialmente las redes sociales han involucionado en poco tiempo. Consideradas, en sus inicios, como el lugar del florecimiento de una nueva esfera pública, un espacio abierto y distribuido de modo no jerárquico, donde el acceso masivo a los medios de edición y publicación debía empoderar a aquellos sin voz y favorecer la diversidad del discurso público. Hoy en día, estas son consideradas como el coadyuvante de una "nueva brutalidad", un espacio some-

tido a la administración de algoritmos que perpetúan las desigualdades y sesgos sociales y donde se llevan a cabo las estrategias de una propaganda política, cuya eficiencia se ha visto amplificada gracias a los recursos del microtargeting y la automatización de la difusión, y cuyas consecuencias son la polarización social y el triunfo del extremismo dentro de los filtros burbuja. Este artículo expone el contexto de esta nueva brutalidad para analizar el papel que desempeñan en la formación del mismo las tecnologías que median los procesos comunicativos y las soluciones propuestas por los diferentes actores implicados en el sistema a la luz de una perspectiva posthumanista.
\end{abstract}

PALABRAS CLAVE

Noticias falsas, Bots, Redes sociales, Administración algorítmica, Desinformación, Posthumanismo

\section{ABSTRACT}

The web and concretely social networks are in fast decay. Hitherto considered the place for the flourishing of a new public sphere, space open and decentralised that allowed a massive access to the publishing media, empowering underdogs and favouring a diverse public discourse. Nowadays, in the epoch of the algorithmic hypermediatization, the web is shifting towards the space of algorithmic bias, and of an amplified and more efficacious politic propaganda, - fostered by the resources of micro-targeting and automatic diffusion, as well as, a space polarized by filter bubbles, where extremism resonates. This decay of the public sphere is becoming a threat to democracy that some authors designate as new brutalism. This article exposes the context of this degradation and analyses the role that technological communication mediations play on it, as well as, the different solutions proposed $y$ the actors involved in this system from the point of view of posthumanism.

\section{KEY WORKS}

Fake news, Bots, Social network, Algorithmic management, Misinformation, Posthumanism mercantilización de la conducta. Hipertext.net, (17), 74-82. DOI:10.31009/hipertext.net.2018.i17.07 


\section{La web en la era de la hipermediación algorítmica: De la esfera pública a la mercantilización de la conducta}

La evolución del sistema técnico nos ha llevado a una situación de mediación radical (Grusin, 2105) o cibernitización de la cultura (Hörl, 2015) que ha modificado nuestro entorno hacia un ecosistema en red y saturado de objetos tecnológicos. En este ecosistema los objetos tecnológicos se convierten en agentes, entidades algorítmicas que median nuestras interacciones dentro y fuera de la red, sin que seamos conscientes de los mismos y de sus procesos. En su texto Hörl expone como esta situación ha modificado los modos de producción de sentido y representación tradicionales, dando lugar a una cultura basada en el control y la regulación. Las entidades estables tradicionales siendo sustituidas por el proceso de retroalimentación de ensamblajes tecnológicos complejos, con los que nos hallamos siempre involucrados, y que son capaces de monitorizar y prever cualquier evento, así como de modificar el entorno. Así mismo, Grusin propone el término "mediación radical" para exponer cómo los medios tecnológicos, no solo operan a nivel epistemológico, como modos de producción de conocimiento, sino que operan a nivel material y afectivo. La mediación no opera de modo neutral reproduciendo y transmitiendo sentidos e información, sino que transforma de modo activo los estados conceptuales y afectivos de humanos y no humanos, de aquí que siempre sea transformadora y desempeñe un papel activo en la producción del entorno.

Este devenir ecológico o infrastructural de los objetos tecnológicos ha modificado nuestros espacios de comunicación, concretamente ha cambiado los mecanismos que regían la comunicación en línea que han evolucionado hacia un progresivo control de nuestra percepción del mundo, y de este modo, de nuestra conducta.

Los pioneros del ciberspacio Pierre Levy (1994) y Manuel Castells (1996) teorizaron la web como nueva esfera pública, el lugar para la comunicación masiva y de un nuevo empoderamiento colectivo, gracias a la accesibilidad colectiva a los medios de publicación y edición. Hoy en día esta esfera pública agoniza bajo el monopolio de las grandes compañías de medios y de la administración algorítmica que gobierna la comunicación en red. Si anteriormente la web había puesto al alcance de todos las herramientas para acceder y contribuir a un conocimiento producido y clasificado colectivamente, hoy en día, nuestras comunicaciones son procesadas, monitorizadas, clasificadas y distribuidas por nuevos agentes inteligentes que operan en un entorno complejo, un nuevo ecosistema que ha puesto en comunicación diversos agentes con distintos propósitos. Usuarios, anunciantes, agencias de medios tradicionales, legisladores e instituciones conviven en un mismo espacio que, bajo el discurso de neutralidad, asociado a la denominación "plataforma", esconde las operaciones algorít- micas y de extracción de datos, que no sólo permiten procesar el aluvión de información que circula por las mismas, sino que las hace económicamente rentables.

La web está sometida a la mediación de algoritmos que filtran y recomponen nuestra percepción del mundo al margen de nuestra consciencia. Un hecho que empeora cuando estos agentes son obscurecidos bajo las leyes de propiedad intelectual que protegen los intereses de los gigantes tecnológicos que han monopolizado la web y cuando el último principio que rige estos procesos de extracción de datos y clasificación, es la maximización del beneficio económico, de un modelo basado en la economía de la atención y en el que el producto son los usuarios. Un modelo en que el entorno es continuamente modificado para ajustarse a las preferencias de los usuarios, con la finalidad de que estos permanezcan pegados a la pantalla, atentos a los anuncios de la misma y produciendo más datos -que a su vez serán utilizados para reajustar el entorno que condiciona nuestra percepción del mundo y nuestras posibilidades de acción-, culminando un proceso en que lo que se mercantiliza es nuestra conducta.

En el proceso de captación de atención y extracción de datos, las plataformas que sostienen la comunicación colectiva llevan a cabo operaciones de perfilado, recomendación y segregación de los usuarios, cuyo efecto secundario más escandaloso es la propagación de noticias falsas y desinformación y su empleo en estrategias de manipulación política.

Si introducimos cualquiera de los términos ingleses "disinformation", "misinformation" o el más periodístico "Fake News" se produce una explosión de contenidos, que van desde acusaciones sensacionalistas - "Fake News" es uno de los términos más utilizados por Donald Trump, para desacreditar a los medios de información tradicionales-, a artículos periodísticos y gran cantidad de estudios en los que nuevas tecnologías y ciencias sociales unen esfuerzos para desentrañar los procesos en que se produce este problema. Este aluvión de entradas indica como la propagación de la falsedad en la red se ha convertido en un problema social, al punto de haber sido considerado por El Fórum Económico Mundial como una de las amenazas que enfrenta la sociedad a nivel mundial.

Para empezar a elucidar qué es y cómo se produce este fenómeno es útil hacer una distinción entre estos términos y ver como cada uno se relaciona con la comunicación social en red. A este fin se expone la descripción propuesta por Jackson (2017).

La desinformación es un término anterior a la era digital, cuyo uso se relaciona con el del término propaganda, diferenciándose del mismo en que éste no busca promover unas ideas concretas, sino que, para llegar al resultado deseado, hace uso de cualquier mensaje efectivo, sin tener en cuenta su veracidad o falsedad, de este modo puede recurrir a mensajes tergiversados y opiniones inflamatorias. "En este tipo de men- 
saje la fuente se mantiene oculta y su finalidad es engendrar cinismo público, incerteza, apatía, desconfianza y paranoia" (Jackson, 2017). Es decir, produce un choque epistemológico, en que la confusión lleva a la falta de acción social.

Las noticias falsas, son un tipo de contenido más directamente relacionado con la web, este se engendra en el modelo de comercialización de los servicios ofrecidos por las redes sociales y esta posibilitado por el acceso a herramientas de creación y propagación de contenidos que ofrecen las mismas. Estas consisten en contenidos hechos a medida para un público-objetivo, identificado por las estrategias de microtargeting que emplea la web social. De este modo estas difieren de la desinformación en que son un fenómeno intrínsecamente relacionado con la economía de la atención y al hecho de que las grandes compañías de Internet extraen sus beneficios de la publicidad. También difieren en que, todo y que pueden tener consecuencias políticas su finalidad también puede ser la búsqueda de un beneficio económico. Este es el caso de los jóvenes de Veles, la población de Macedonia en que un grupo de adolescentes se convirtieron en promotores de la campaña de Donald Trump, creando una serie de webs con contenidos falsos que se publicaban en Facebook con la finalidad de recaudar dinero mediante el sistema de 'pay per click' del servicio de Google AdSense. (Subramanian, 2017).

Finalmente, el término inglés 'misinformation', que podríamos traducir como falta de información, es un término explícitamente relacionado con la comunicación en red y hace referencia a la tendencia de los usuarios a compartir contenidos falsos de modo inadvertido.

El informe sobre manipulación de los medios realizado por la organización Data and Society concluye que la desinformación puede contribuir al aumento de la desconfianza en los medios tradicionales, el incremento de la circulación de hechos falsos y en un ulterior incremento de la radicalización (Marwick y Lewis, 2017).

Esta degradación de la esfera pública es un fenómeno complejo que afecta a todo el ecosistema informativo y cuyos orígenes se hayan en problemas sociales y políticos que han contribuido a la pérdida de confianza en las instituciones que otrora habían funcionado como garantes de la veracidad, entre estos tanto los medios de comunicación tradicionales como las instituciones gubernamentales y culturales. El análisis mostrado en este artículo, todo y que se contextualiza en la realidad política actual, se centra en el funcionamiento de las nuevas tecnologías aplicadas a la comunicación en red. Concretamente como la hipermediación algorítmica - término aquí propuesto para hacer referencia a los procesos de extracción de datos, clasificación y difusión de contenidos mediante el uso de algoritmos - han contribuido a la aparición de nuevos procesos y agentes, entre ellos los filtros burbuja, el sesgo algorítmico y los bots políticos, que se entretejen en la comunicación social, modificando el espacio de discurso público hacia una crisis de la cultura participativa (Lovink, 2017), la precarización de la democracia y la emergencia de una nueva brutalidad (Braidotti, et al., 2017).

\section{La evolución del Sistema Sociotécnico. Nuevos agentes y el filtrado "inteligente" de contenidos}

En 2008 el uso de técnicas de microtargeting y crowdsourcing en la campaña de Barack Obama pusieron a las nuevas tecnologías que comandan las redes, como aliados estratégicos en la consecución de un hito histórico. Por primera vez un afroamericano ocupaba la presidencia de Estados Unidos. Este hecho fue seguido por la primavera árabe en 2011, en que el uso extensivo, por parte de la sociedad civil, de las redes sociales y la comunicación móvil, llevarían a derrocar al dictador Mubarak. Sin embargo, poco después estas mismas herramientas serían adoptadas por los poderes establecidos, la asamblea nacional, que sustituyó al dictador egipcio no tardo en crear su propia página en Facebook y canalizar todas sus comunicaciones desde ese lugar, contrarrestando las publicaciones de los disidentes. La alarma saltó en 2016 cuando Mark Zuckerberg, fundador de Facebook, tuvo que hacer frente a las acusaciones que señalaban a la plataforma como responsable de influenciar el resultado de las elecciones americanas. Donald Trump hizo un uso extensivo de las herramientas de microtargeting y difusión automatizada de plataformas como Facebook y Twitter, supuestamente apoyado en los servicios de la compañía Cambridge Analytica, creando un ambiente de desinformación en el que también participaron seguidores y oportunistas en busca de beneficio económico.

Estas mismas herramientas se aplicaron a la campaña del Brexit que llevaría al Reino Unido a escindirse de Europa. Desde entonces la desinformación que se propaga a través de las redes sociales se ha asociado al mantenimiento del conflicto en Siria (Denselow, 2017), La promoción de sentimientos nacionalistas por parte del movimiento 5 Estrellas en Italia (Nadelli y Silverman, 2016), la difusión de sentimientos de odio y la limpieza étnica de la minoría Rohingya en Myanmar y de la injerencia de hackers rusos, como el grupo Fancy Bear, en las elecciones de distintos países europeos, con la finalidad de destruir la estabilidad y cohesión europea. Este nuevo aparato de propaganda altamente tecnológico y embebido en la web no es un hecho aislado en Rusia, un reciente informe realizado por el Instituto de Internet de la Universidad de Oxford ha identificado la presencia de "cyber troops" - grupos gubernamentales o pertenecientes a partidos políticos dedicados a manipular la opinión pública en las redes sociales - en 28 países (Bradshaw y Howard, 2017).

El uso de las redes sociales como arma política ha llevado a lo que algunos teóricos califican de nueva brutalidad (Braidotti, et al., 2017), El informe sobre la salud de Internet, publicado anualmente por Mozilla, atribuye este uso político de las redes mediante la proliferación de noticias falsas, campañas 
de astroturf, acoso a minorías y el hackeo de la atención por parte de grupos extremistas a que "la economía de la publicidad en línea que subyace actualmente a la mayoría de servicios de Internet esta terriblemente rota" (Mozilla Foundation, 2018). Un hecho que se ve agravado por el monopolio que ejercen unas pocas compañías sobre la comunicación en línea y la falta de alfabetización digital, en parte debida a la preeminencia de servicios listos para su uso que ocultan su funcionamiento al usuario. Actualmente Facebook ha alcanzado 2 billones de usuarios, y Google recibe alrededor de 3 billones de búsquedas diarias, ambas compañías son responsables de canalizar un $70 \%$ del tráfico que se genera en la web. La preeminencia de estos medios, el hecho de que trabajan coordinadamente entre ellos y otros servicios de su propiedad, sumado al triunfo de la conectividad móvil, que hace no solo que estemos permanentemente conectados y generando datos, sino que interactuemos con la web mediante los servicios que ofrecen estas compañías, ha convertido a estos gigantes tecnológicos no solo en agentes activos en la producción de discurso social, sino en mediadores permanentes de nuestra percepción del entorno.

Facebook y Google aparecieron en el cambio de siglo, Google en 1995, como una innovadora alternativa a los buscadores tradicionales y Facebook en 2004 siguiendo el modelo de anteriores redes sociales. Un servicio que permitía al usuario crearse una identidad digital, descrita en un perfil y entrar en contacto con usuarios con intereses similares. Ambos proveedores de servicios se consideran modelos paradigmáticos de la denominada web 2.0, "la comunicación en línea basada en la habilidad de las personas para usar una serie de tecnologías de información y comunicación ofrecidas por una plataforma, para expresarse y participar en el espacio común generado por el ciberespacio" (Gillespie, 2010). Gillespie recalca como el término plataforma no emerge en el discurso que promueve estos medios de comunicación para hacer sólo referencia a una arquitectura funcional, sino que "esta sugiere disposiciones igualitarias y progresivas que aventajan a aquellos que se sitúan en ella" (Gillespie, 2010). De este modo, el término plataforma conlleva connotaciones que califican a los entornos producidos por los proveedores de servicios en linea como abiertos, neutrales, igualitarios y progresivos. Un entorno que empodera a sus usuarios a hablar y participar en el discurso democrático, enfatizando el contenido generado por el público, la creatividad popular, el contacto social entre pares y el comentario robusto. De este modo estos servicios se promueven tras las misiones altruistas de estos medios. Google empeñado en "organizar el conocimiento del mundo y hacerlo universalmente accesible y utilizable" (Google,2018), Facebook en "Hacer el mundo más abierto y conectado" (Zuckerberg, 2015), al mismo tiempo, que se mantienen ajenos a la responsabilidad respecto a los contenidos que circulan a través de los mismos y ocultan las maniobras algorítmicas que bloquean contenidos ofensivos o que infringen las leyes del copyright. Esta neutralidad entra en conflicto con el sistema económico en que se sustentan estos servicios. El termino plataforma al mismo tiempo connota oportunidad. Las plataformas empoderan a los anunciantes facilitándoles datos que les permiten comprender y conectar con la audiencia, controlar el contenido y tomar decisiones inteligentes. De este modo se produce una paradoja en que "propiciar el contenido generado por los usuarios y empoderar a los individuos, depende de un modelo de negocio basado en la publicidad y en el que los usuarios son vendidos a los anunciantes" (Gillespie, 2010).

El interés económico ha llevado a estas empresas a acumular cada vez más datos, mediante la adquisición de nuevos servicios - en el caso de Google mediante la creación de Gmail en 2004, Blogger en 2003, Picassa en 2004, servicios cartográficos y de localización geográfica con la adquisición de Keyhole Inc. en 2004 y la posterior creación de Google Maps... Por su parte Facebook adquirió Messenger en 2014, servicio de mensajería instantánea que se uniría a Whatsapp y a la red social, basada en fotografías, Instagram - o la prestación de servicios a terceros, botones de like o compartir y servicios de login que se distribuyen en la red creando un ecosistema conectado, que permite a estas corporaciones rastrear las acciones de sus usuarios a través de la web.

El modelo de plataforma produce una explosión de contenido y con ello, la necesidad de la implementación de algoritmos que permitan extraer datos y administrar estos contenidos de modo eficiente. Gillespie explica como los algoritmos que operan sobre un corpus de datos compuesto por los trazos de nuestras actividades, preferencias y expresiones tienen relevancia pública (Gillespie 2014). Estos no solo ayudan a encontrar información, sino que modelan nuestra percepción y procesos de conocimiento. Este autor destaca seis dimensiones a través de las cuales los algoritmos operan en el discurso social y político. Los procesos de colección y formalización de datos crean patrones de inclusión, que determinan que es susceptible de ser un contenido computable y por tanto ser distribuido por la plataforma. También crean ciclos de anticipación, sometiendo a sus usuarios a procesos de vigilancia y extrayendo conclusiones a partir de categorías parciales y pobremente definidas. Categorías como edad, situación, familiar, tendencias políticas, aficiones, nivel cultural, ocupación profesional ... se definen a partir de los datos generados en nuestras interacciones, como los dispositivos y lugares desde los que nos conectamos, amigos con los que interactuamos o contenidos que compartimos. Estas son empleadas en la definición de un perfil que ayudan a los anunciantes a saber cuáles son nuestros hábitos y preferencias, si podemos estar interesados en sus productos y si somos objetivos deseables. Una tercera dimensión de los algoritmos evalúa la relevancia de los contenidos en base a criterios de eficiencia y premisas epistemológicas ocultas. Estas acciones operan bajo una cuarta dimensión la premisa de objetividad, que mantiene estos procesos al margen de procesos de enjuiciamiento, mientras se implican en la práctica y producen públicos calcu- 
lados, últimas dos dimensiones. Estas dimensiones implican los algoritmos en la emergencia de procesos y agentes que median el discurso público y modifican el entorno comunicativo, poniéndolo a disposición, no solo de estrategias de promoción y venta sino de la manipulación política. Agentes maliciosos se ven capacitados para crear mensajes a medida y destinados a ciertos grupos para promover o desacreditar líderes políticos o instigar el odio hacia determinadas minorías.

La implicación en la práctica y la creación de públicos calculados tienen incidencia en la producción de filtros burbuja. Con el objetivo de mantener nuestra atención e incrementar el tiempo que pasamos en la plataforma, Facebook elimina las historias redundantes o aburridas y privilegia aquellas publicaciones que conforme al registro de nuestra actividad son más propensas a generar una reacción. Publicaciones que nos aproximan a nuestros contactos cercanos y nos resultan atractivas conforme a nuestras preferencias e ideología. Esto genera una ordenación homofílica en que somos segregados en grupos cerrados y solo en contacto con aquellos que piensan como nosotros, un entorno homogéneo y hecho a medida que funciona como una cámara de resonancia que amplifica nuestros prejuicios, gracias a la tendencia que tenemos a considerar como verdaderas aquellas informaciones que confirman nuestras creencias (Nyhan y Reifler, 2010; Weeks, 2015). De acuerdo con Eli Pariser - activista y autor del Libro "Filter Bubble", que popularizo el término filtro burbuja en 2011- la democracia sólo funciona si los ciudadanos son capaces de pensar más allá del estrecho campo definido por sus propios intereses. Para que esto se produzca, necesitamos una visión compartida del mundo en el que cohabitamos. Los filtros burbuja nos empujan en la dirección contraria, creando la impresión de que aquello que cae bajo nuestro interés personal es lo único que existe. Aquello que hizo de la web el lugar de encuentro de la sociedad civil silenciada, el hecho de que en Internet nadie está solo, posibilitando que reivindicaciones legitimas avanzaran hacia una masa crítica capaz de impulsar el cambio social, una vez sometido a la eficiencia y objetividad de la administración algorítmica nos ha apartado de la diversidad de puntos de vista que posibilitan el discurso democrático y el surgimiento de propuestas colectivas legitimas. En este ambiente los recursos de contra narrativa como memes anteriormente utilizados como instrumentos de crítica social, se convierten en medios para reforzar ideologías dentro de las burbujas. "No nos hallamos perdidos en un laberinto, sino más bien arrojados al vacío, vigilados y manipulados, sin ningún centro de comando a la vista" (Lovink, 2017).

La clasificación y difusión algorítmica de los contenidos de Internet crea otros problemas. Google quien ejerce maniobras de microtargeting en su servicio de anuncios Adsense, también se ha visto implicado en la fabricación y difusión de noticias falsas, además de verse envuelto en la reproducción de sesgo algorítmico al aplicar algoritmos de Machine Lear- ning a su sistema de autocompletado en la caja de búsqueda y en la priorización de resultados. Este procedimiento da forma al vocabulario social, privilegiando ideas y posiciones a expensas de la marginalización de grupos y puntos de vista.

Finalmente nos encontramos a otro agente algorítmico susceptible de manipular el discurso público. Los bots, un bot es un programa que se emplea en Internet para llevar a cabo acciones automatizadas como recabar datos. En las plataformas sociales estos pueden emplearse para publicar actualizaciones periódicas respecto a un hecho concreto, por ejemplo, la información meteorológica. Algunos bots son más sofisticados, implementando inteligencia artificial que los convierte en agentes capaces de observar y actuar en un entorno computacional cerrado para alcanzar fines predefinidos. Estos programas pueden funcionar como amplificadores artificiales de los contenidos diseminados en la red con fines políticos (Gorwa y Guilbeault, 2018) y ser usados en campañas de astroturf con fines políticos. - El astroturf es el proceso de buscar la victoria electoral y cambio legislativo por medio de ayudar a los actores políticos a encontrar y movilizar una masa de público simpatizante, un proceso destinado a crear imagen de consenso cuando no existe (Howard, Woolley y Calo, 2018).

Un reciente estudio efectuado por el Observatorio de Medios Sociales de la Universidad de Indiana muestra que los bots son responsables de la difusión de una parte importante de las noticias falsas que se difunden en la red social Twitter (Shao, et al., 2018). Comparando la actividad generada por una serie de cuentas identificadas por ser productores habituales de contenidos falsos y comparándola con la actividad generada entrono a contenidos verificados por agencias de fact-checking, el estudio concluye que los bots desarrollan una actividad de amplificación importante en los primeros momentos de difusión de un hecho falso, justo antes de que este se haga viral. Centrándose en la actividad de amplificación centrándose en unas cuantas cuentas que pueden llegar a retuitear el mismo hecho cientos de veces. Esta amplificación se ve reforzada por una segunda estrategia consistente en la identificación de usuarios con gran número de seguidores y la captación de su atención mediante la publicación de réplicas y menciones, a la espera de que el usuario comparta el contenido fraudulento. El estudio demuestra que los humanos retuitean más que los bots y retuitean igual mensajes compartidos por cuentas verídicas que falsas, convirtiéndose de este modo en colaboradores involuntarios de la amplificación de noticias falsas. El estudio concluye que la detección y eliminación de bots contribuiría notablemente a mitigar la desinformación.

\section{Investigación y soluciones}

La diseminación de noticias falsas ha generado un gran debate sobre que actores deben implicarse en el control de los contenidos que se distribuyen en las redes y que soluciones 
deben adoptarse. A continuación, se muestran varias propuestas de soluciones entre estas las propuestas por las propias plataformas, mediante la mejora de sus sistemas de administración algorítmica y las propuestas de varios centros de investigación, de las que solo se muestran algunos ejemplos, estas divididas en dos procedimientos. La clasificación y análisis de los procesos implicados en la desinformación destinado a posibilitar una regulación desde arriba, ejercida por instituciones gubernamentales y la propuesta de herramientas y el diseño de experiencias que permitan a los usuarios tomar conciencia y control del flujo de información al que son sometidos.

Después de la denuncia masiva en 2016, las plataformas sociales se han visto forzadas a reconocer que no son meras plataformas en que los contenidos son creados por los usuarios y administrados por algoritmos neutrales. En una nota publicada en Facebook el 16 de febrero titulada "Construyendo la Comunidad Global" (Zuckerberg, 2016) Zuckerberg admitía el papel que la plataforma juega en la construcción de discurso público y la comprometía al esfuerzo de amplificar los efectos positivos de la misma y eliminar los negativos. A esta declaración le seguiría una actualización de la política de seguridad y publicación de la plataforma y el compromiso de poner medios para la detección y eliminación de contenidos perjudiciales, recurriendo a la ayuda de agencias de fact-checking externas (Mosseri, 2016). Desde entonces, Facebook ha extremado las medidas de seguridad y verificación para evitar el hackeo de cuentas de usuarios y la creación de cuenta falsas, trabaja en sistemas de reconocimiento del contenido, utilizando algoritmos de procesado de lenguaje natural y de reconocimiento de imágenes, para eliminar o disminuir la presencia de contenido susceptible de ser nocivo o de tergiversar la realidad, trabaja para interrumpir los beneficios económicos de los productores de hechos falsos y crea nuevos productos que favorezcan la diversificación de puntos de vista y ayuden a la gente a tomar decisiones más informadas. Estas medidas también son implementadas por Google, quien ha actualizado su política de publicación y prohibido el uso de su plataforma AdSense a los creadores de contenidos falsos, algunas de estas medidas recorren a la ayuda de los usuarios. Por ejemplo, Google ha incluido el botón "report inappropriate predictions" en su página de búsqueda para recabar datos que ayuden a eliminar el sesgo de su algoritmo de autocompletado. También ha elaborado el experimento Perspective (www.perspectiveapi.com) un instrumento que permite a los usuarios calificar un contenido como tóxico y contribuir a una base de datos, que posteriormente es utilizada para entrenar algoritmos de conocimiento máquina (ML), que después serán destinados para para la identificación y filtrado de esos contenidos tóxicos. Twitter también está probando un sistema que permita a los usuarios marcar posts con contenidos falsos, para su posterior discusión. Estas soluciones no salen del sistema y de sus paradojas inherentes, siendo un intento de reparación del mismo en que se mejora los mismos algoritmos que crean el problema mediante el uso de más datos. En otro sentido se dirige el cambio de misión de la compañía anunciado por Zuckenberg en la primera cima de comunidades de Facebook celebrada en Chicago el 22 de junio de 2017. El fundador de la plataforma declama que "no es suficiente con conectar el mundo, también debemos trabajar para acercar a la población mundial" (Zuckerberg, 2017) y propone una serie de herramientas destinadas a propiciar la intervención humana en la plataforma y la creación y dinamización de comunidades significativas.

A los esfuerzos llevados a cabo por las plataformas se une la promoción de legislación que regule la actividad de estos medios, que hasta recientemente habían actuado de forma desreguladora debido a la falta de reconocimiento y definición de su especificad y capacidad de influencia social. En este sentido, el Instituto de Internet de la Universidad de Oxford desarrolla el programa de investigación Propaganda Computacional (www.politicalbots.org). Este investiga las interacciones entre los algoritmos, la automatización y la política, analizando como los bots políticos son utilizados para manipular la opinión pública. Este proyecto combina los puntos de vista de la sociología organizacional, la interacción hombre máquina, las ciencias de la comunicación y de la información y las ciencias políticas para clasificar estas interacciones y desarrollar recomendaciones que puedan contribuir a su regulación por parte de las instituciones responsables.

Más allá del establecimiento de medidas de auto-regulación por parte de las grandes compañías y de la proclamación de leyes y regulaciones institucionales, la comunidad científica también explora medios que pongan herramientas en manos de los usuarios, que les permita mejorar la comprensión de los mecanismos algorítmicos que operan en las redes y tomar medidas para mejorar la comunicación social.

El Observatorio de los Medios Sociales (OsoMe) de la Universidad de Indiana utiliza ciencia de redes y conocimiento de maquina (ML) para modelar la difusión de noticias falsas, la creación de burbujas y polarización en las redes e identificar la presencia de bots. El análisis de la red generada por las interacciones de Twitter permite rastrear la conducta de los usuarios y la difusión de la información dentro de la misma, gracias a la presencia de hastags, retuits, menciones etc. El conocimiento de esta topología es empleado en la creación de herramientas que permitan identificar contenidos falsos y bots en la red. Es el caso de Hoaxy (https://hoaxy.iuni.iu.edu/), este experimento combina conocimiento supervisado - un tipo de ML- basado en características extraídas de la topología de la difusión en red, análisis del sentimiento y anotaciones colectivas para identificar contenidos falsos.

El Botometer (https://botometer.iuni.iu.edu/\#!/) es otro experimento de este laboratorio, este permite comprobar la actividad de una cuenta de Twitter y cuantificar la probabilidad de que se trate de una cuenta operada por un bot. Una 
vez obtenidas las puntuaciones de sus seguidores, el usuario puede eliminar las cuentas sospechosas.

Finalmente, encontramos el diseño de experiencias de usuario que otorgan a los mismos una mayor conciencia de como la hipermediación algorítmica se entreteje y da forma al espacio social, plateando el uso de los medios de comunicación en línea de modo más crítico. El Laboratorio Social Machines del MIT Media Lab, utiliza técnicas como el procesado de lenguaje natural (NLP), la ciencia de redes, el conocimiento de máquina $(\mathrm{ML})$ y el diseño de experiencias de usuario para crear experimentos que promuevan una mejor comprensión de las redes y el entorno comunicativo que estas generan. Entre estos podemos citar el FlipFed (https://www.media.mit.edu/projects/ flipfeed/overview/), una extensión del buscador Crome que nos permite intercambiar nuestro muro con el de otro usuario real de Twitter. Haciendo uso de algoritmos de deep learning y análisis de redes, el programa reconoce las tendencias políticas del usuario y remplaza su feed por el de un usuario con tendencias contrarias, de este modo el usuario es expuesto a historias y comentarios completamente distintos a los que el suele consumir, ampliando su perspectiva del entorno comunicativo y escapando a la polarización ideológica que se produce en las redes, lo que le da la oportunidad de empatizar con otros puntos de vista.

Otro experimento es el Social Media Mirror, una aplicación web que permite a los usuarios de Twitter explorar de modo interactivo las partes políticamente activas de su red. Una forma de auto-reflexión que pretende inspirar a los usuarios a buscar conexiones fuera de su red inmediata mitigando la polarización.

Finalmente, Gobo ( https://www.media.mit.edu/projects/ gobo/overview/Gobo) es un agregador de medios sociales que incluye una serie de filtros, que pueden ser controlados por el usuario. Estos controles permiten explorar las publicaciones de nuestro muro, señalando los posts que son mostrados y los que quedan excluidos por los ajustes seleccionados en nuestros filtros. Un modo de ser conscientes de las acciones de filtrado de las plataformas y de devolver el control de sus muros a los usuarios.

Estos proyectos están dirigidos a contribuir a la regulación de la comunicación hipermediatizada y a proponer un uso más consciente de los medios de comunicación en red, sin embargo, estos no llegan a interrumpir el funcionamiento del sistema ni a proponer una alfabetización crítica de los mismos. Estos proyectos hacen uso de las mismas mediaciones, objetos y metodologías como el diseño de interfaces o modelado de redes, implicadas en esta hipermediación, para proponer herramientas y experiencias alternativas, pero quedan circunscritos al uso y conocimiento de una élite académica y no desvelan o hacen extensivos los procesos y mecanismos de la misma. Las redes sociales, buscadores y demás plataformas que constituyen la web se han embebido en nuestras vidas, cambiando el modo en que nos conducimos en la vida diaria y promoviendo modos de conducta, acción y conocimiento. A pesar de su acción disruptiva en la esfera pública, estos también han aumentado nuestras capacidades de conocimiento conectándonos y ampliando nuestro mundo, por lo que no parece factible el renunciar a los mismos, sin embargo, es necesario promover medios que nos permitan ganar capacidad de juicio y control sobre sus mediaciones.

\section{Conclusión: Hacia una ética posthumanista}

Rosi Braidotti señala la era de la posverdad como la era de una nueva brutalidad en que técnicas reprobables como el engaño el bulling y la instigación del racismo y la xenofobia se han convertido en herramientas de la construcción del discurso político (Braidotti, et al., 2017). Ahora marcado por la disolución de la esfera pública y la pérdida de todos sus privilegios, en favor de la imposición de perspectivas de suma-cero y la destrucción de las aportaciones a la subjetividad del pensamiento posmoderno. Una situación en que la sociedad civil se halla fracturada en piezas, e incapacitada para actuar, mientras el parlamentarismo representativo es sustituido por el populismo. Ante esta situación, la autora promueve una ética posthumanista de inspiración Spinozista, una ética relacional en que somos conscientes de nuestra situación en el mundo y de las relaciones que mantenemos con todos los agentes humanos y no humanos que lo pueblan y cuyo instrumento es un pensamiento crítico acompañado de creatividad (Braidotti, 2006). Seguidora de las propuestas del neo materialismo feminista, el ejercicio de esta ética pasaría por la creación de dispositivos que encarnaran las mediaciones que rigen la comunicación en linea y promovieran el conocimiento de los mismos, al mismo tiempo, que alternativas en que la relación con las nuevas tecnologías fuera más consciente, e inclusiva.

La decadencia de la esfera pública es un hecho complejo cuya solución no puede dejarse solo en manos de los recursos tecnológicos de los gigantes tecnológicos que gobiernan Internet ni en una legislación impuesta desde arriba por instituciones alejadas de los hechos y las problemáticas diarias. Esta requiere de la participación de todos los implicados y del desarrollo de herramientas tecnológicas o tradicionales, pero siempre creativas que ayuden a la comprensión y una participación activa en las mediaciones que conforman la representación y la acción en nuestro mundo.

\section{Referencias}

Bradshaw, S. y Howard, P. (2017). Troop, Trolls and Troublemakers: A global inventory of Organized Social Media manipulation. (Working Paper no 2117.12 ). Computational Propaganda Research Project. Recuperado de http://comprop.oii.ox.ac.uk/wp-content/uploads/ sites/89/2017/07/Troops-Trolls-and-Troublemakers.pdf

Braidotti (2006). Affirmation versus Vulnerability: On Contemporary Ethical Debates. Canadian Journal of Continental Philosophy, 10, (1), 


\section{5-254.}

Braidotti, R., Vermeulen, T, Aranda, J., Kuan Wood, B., Squibb, S. y Vidokle, A. (2017) The New Brutality. e-flux journal (\#83). Recuperado en https://www.e-flux.com/journal/83

Castells, M. (1996). The Rise of Network Society. The Information Age: Economy, Society and Culture. Vol. I. Cambridge, Massachusetts; Blackwell.

Denselow, J. (2016). Syria's civil war is a post-factual conflict. Aljazeera, August 3, 2016. Recuperado de https://www.aljazeera. com/indepth/opinion/2016/08/syria-civil-war-post-factual-conflict-160803075226372.html

Gillespie, T. (2010). The politics of "platforms. New Media \& Society, 12(3), 347-364.

Gillespie, T. (2014). The Relevance of Algorithms. En T. Gillespie, P. Boczkowski, y Kirsten Foot (ed), Media Technologies. (pp. 167-194). Cambridge, Massachusetts: Mit University Press Group Ltd.

Gomes, B. (2017) Our latest quality improvements for search. Google Blog. Recuperado de https://www.blog.google/products/search/ our-latest-quality-improvements-search

Google (2018). About. Recuperado de https://www.google.com/about

Gorwa, R., y Guilbeault, D. (2018). Unpacking the Social Media Bot: A Typology to Guide Research and Policy. Policy \& Internet. DOI: 10.1002/poi3.184

Grusin, R. (2015). Radical Mediation. Critical Inquiry, 42(1), 124-148.

Hörl, H. (2015). The technological Condition. Parrhesia, (22), 1-15.

Howard, P.N., Woolley, S., y Calo, R. (2018). Algorithms, bots, and political communication in the US 2016 election: The challenge of automated political communication for election law and administration. Journal of Information Technology and Politics, 15(2), 81-93.

Jackson, D. (2017). Distinguishing Disinformation from Propaganda, Misinformation, and "Fake News". National Endowment for Democracy, 17 october 2017. Recuperado de https://www.ned.org/ issue-brief-distinguishing-disinformation-from-propaganda-misinformation-and-fake-news

Levy, P. (1994). L'Intelligence collective. Pour une anthropologie du cyberespace. Paris: La Découverte.

Lovink, G. (2017). Overcoming Internet Disillusionment: On the Principles of Meme Design. e-flux journal, (\#83). Recuperado de https:// www.e-flux.com/journal/83

Marwick, A. y Lewis, R. (2017). Media Manipulation and desinformation. Data \& Society. Recuperado de https://datasociety.net/output/ media-manipulation-and-disinfo-online

Mosseri, A. (2016). Addressing Hoaxes and Fake News. Facebook News Room, December 15, 2016. Recuperado de https://newsroom. fb.com/news/2016/12/news-feed-fyi-addressing-hoaxes-and-fakenews

Mozilla Fundation (2018). Internet Health report. Recuperado de https://internethealthreport.org

Nadelli, A., y Silverman, C. (2016). Italy's Most Popular Political Party Is Leading Europe In Fake News And Kremlin Propaganda. BuzzFedd, November 29, 2016. Recuperado de https://www.buzzfeed.com/ albertonardelli/italys-most-popular-political-party-is-leading-europe-in-fak?utm_term=.wkY3JLRqO\#.tjoNd7m31

Nyhan, B., y Reifler, J. (2010). When Corrections Fail: The Persistence of Political Misperceptions. Political Behavior, 32(2), 303-330.
Ratkiewicz,J., Conover, M.D., Meiss, M., Gonçalves, B., Flammini, A. y Menczer, F. (2011). Detecting and Tracking Political Abuse in Social Media. Proceedings of the 5th AAAl International Conference on Weblogs and Social Media (ICWSM'11).

Shao C, Hui P-M, Wang L, JiangX, Flammini A, Menczer F, et al. (2018). Anatomy of an online misinformation network. PloS ONE, 13(4): e0196087. Recuperado de https://doi.org/10.1371/journal. pone. 0196087

Stamos A. (2017). An update on information operations on facebook. Facebook newsroom, September 6, 2017. Recuperado de https:// newsroom.fb.com/news/2017/09/information-operations-update

Subramanian, S. (2017). Inside the macedonian fake-news complex. Wired, February 15,2017. Recuperado de https://www.wired. com/2017/02/veles-macedonia-fake-news

Weeks, B. E. (2015). Emotions, Partisanship, and Misperceptions: How Anger and Anxiety Moderate the Effect of Partisan Bias on Susceptibility to Political Misinformation. The Journal of Communication, 65(4), 699-719.

Weedon, j., Nuland, W. y Stamos, A. (2017). Information operations and facebook". Facebook newsroom, April 27, 2017. Recuperado de https://fbnewsroomus.files.wordpress.com/2017/04/facebook-and-information-operations-v1.pdf

Zuckerberg, M. (2015). Post. Facebook Notes, December 22, 2015. Recuperado de https://www.facebook.com/zuck/ posts/10102542090197661

Zuckerberg, M. (2016). Building Global Community. Facebook Notes, February 16, 2017. Recuperado de https://www.facebook.com/notes/ mark-zuckerberg/building-global-community/10154544292806634

Zuckerberg, M. (2017). Post. Facebook Notes, June 22, 2017. Recuperado de https://www.facebook.com/zuck/posts/10154944663901634

\section{CV}

Sandra Álvaro. Es doctora europea en filosofía por la Universidad Autónoma de Barcelona. En el desarrollo de su investigación ha sido investigadora-becaria en el Instituto para las Matemáticas Puras y Aplicadas (IPAM) de la Universidad de UCLA, investigadora/artista invitada en el laboratorio Paragraphe/CITU de la Universidad París-8, y ha ejercido como docente en el Estudio Roy Ascott de arte y tecnología del DeTao Masters Academy de Shanghái. Ha publicado en revistas científicas como el Technoetics Art Journal (Intellect) y Artnodes y ha participado en númerosos congresos y simposios internacionales, entre otros, el "Arts Incognita. Poetics of Artificial creativity" (Cordoba, 2018), Open Fields Festival (Riga, 2017), CAC (París, 2016), ISEA (Vancouver, 2015), Re-new (Copenhague, 2013) y el CR13 International Research Conference in the series of Consciousness Reframed: Art and Consciousness in the post-biological era (El Cairo, 2013). También es escritora independiente y productora cultural. www.sandraalvaro.net 


\section{MÁSTER UNIVERSITARIO ONLINE EN OCUMENTACIÓN DIGITAL} DISEÑA, CREA, GESTIONA, EVALÚA, COMPARTE Y PROMOCIONA RECURSOS DIGITALES

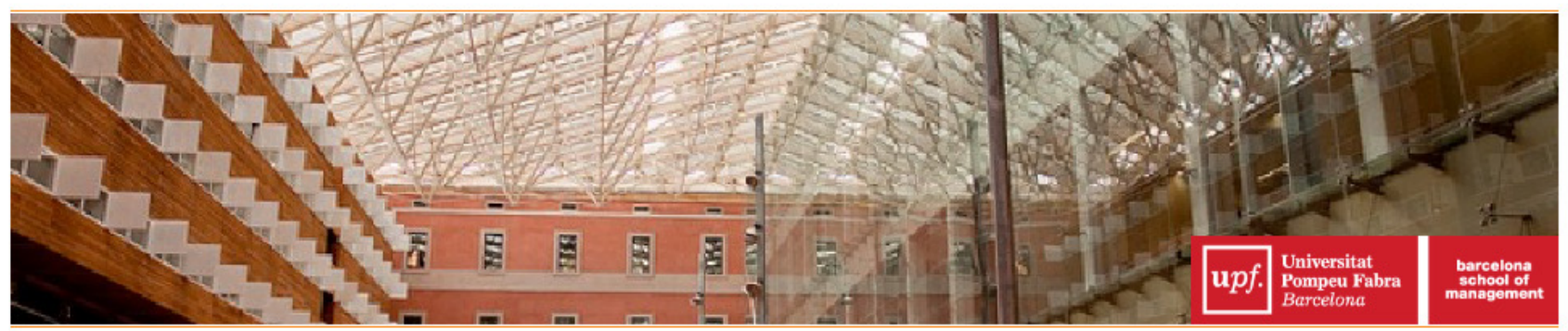

\section{MASTER EN USABILIDAD, DISEÑO DE INTERACCIÓN Y EXPERIENCIA DE USUARIO (ONLINE)}

MÁSTER EN UX

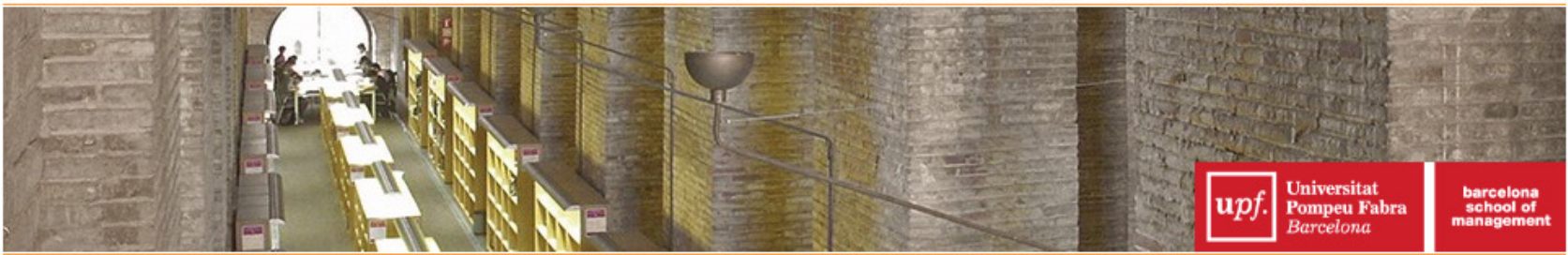

\section{MÁSTER EN BUSCADORES}

MARKETING (SEM) · POSICIONAMIENTO (SEO) . ANALYTICS . SOCIAL MEDIA

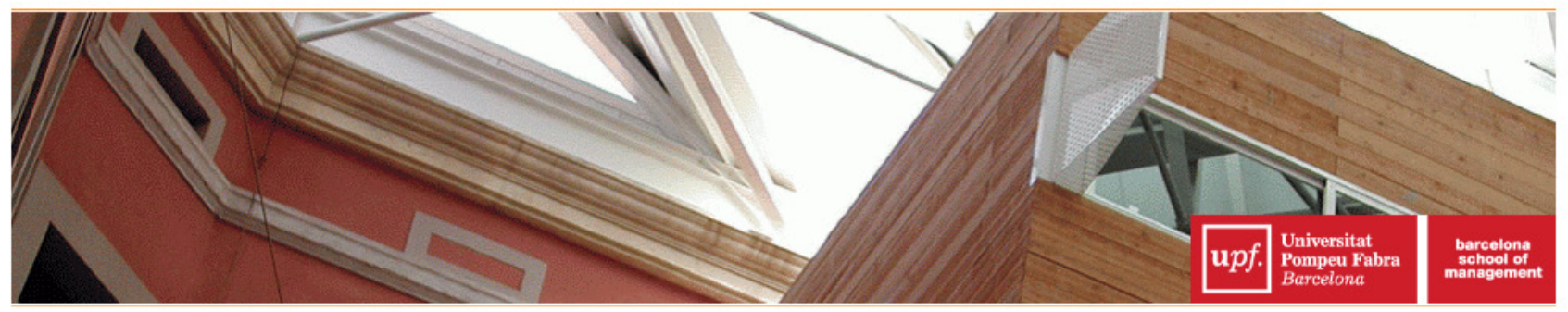

\section{OCM}

\section{Observatorio deCibermedios}

https://observatoriocibermedios.upf.edu/

\begin{tabular}{|l|ll}
$\mathbf{u} p \boldsymbol{p}$. & $\begin{array}{l}\text { Universitat } \\
\text { Pompeu Fabra } \\
\text { Barcelona }\end{array}$ & $\begin{array}{l}\text { Departamento } \\
\text { de Comunicación } \\
\text { Grupo DigiDoc }\end{array}$ \\
\cline { 1 - 2 } &
\end{tabular}

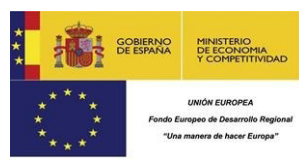

El Observatorio de Cibermedios es una producción del Grupo de Investigación en Documentación Digital y Comunicación Interactiva (DigiDoc) del Departamento de Comunicación de la Universitat Pompeu Fabra.

El Observatorio de Cibermedios (OCM) forma parte del proyecto del Plan Nacional "Creación y contenido interactivo en la comunicación de información audiovisual: audiencias, diseño, sistemas y formatos". CS02015-64955-C4-2-R (MINECO/ FEDER), Ministerio de Economía y Competitividad (España). 